\title{
Optical fiber interferometer array for scanless Fourier-transform spectroscopy
}

\author{
Aitor V. Velasco, ${ }^{1, *}$ Pavel Cheben,,${ }^{2,4}$ Mirosław Florjańczyk, ${ }^{2}$ Jens H. Schmid, ${ }^{2}$ Przemek J. Bock, ${ }^{2}$ Jean Lapointe, ${ }^{2}$ \\ André Delâge, ${ }^{2}$ Siegfried Janz, ${ }^{2}$ Martin Vachon, ${ }^{2}$ María L. Calvo, ${ }^{1}$ Dan-Xia Xu, ${ }^{2}$ and Svatopluk Civiš ${ }^{3}$ \\ ${ }^{1}$ Departamento de Óptica, Facultad de Ciencias Físicas, Universidad Complutense de Madrid, Madrid 28040, Spain \\ ${ }^{2}$ National Research Council Canada, Ottawa, Ontario K1A OR6, Canada \\ ${ }^{3}$ J. Heyrovský Institute of Physical Chemistry, Academy of Sciences of the Czech Republic, 18223 Prague 8, Czech Republic \\ ${ }^{4} e$-mail: pavel.cheben@nrc.ca \\ *Corresponding author: avillafr@pdi.ucm.es
}

Received March 22, 2013; revised May 14, 2013; accepted May 24, 2013; posted May 24, 2013 (Doc. ID 187599); published June 25, 2013

\begin{abstract}
We report a spatial heterodyne Fourier-transform spectrometer implemented with an array of optical fiber interferometers. This configuration generates a wavelength-dependent stationary interferogram from which the input spectrum is retrieved in a single shot without scanning elements. Furthermore, fabrication and experimental deviations from the ideal behavior of the device are corrected by spectral inversion algorithms. The spectral resolution of our system can be readily scaled up by incorporating longer optical fiber delays, providing a pathway toward surpassing current spectroscopy resolution limits. (c) 2013 Optical Society of America

OCIS codes: (060.2340) Fiber optics components; (300.6300) Spectroscopy, Fourier transforms.

http://dx.doi.org/10.1364/OL.38.002262
\end{abstract}

Fourier-transform (FT) spectrometry [1] is an elegant and practical realization of the principle of Fourier decomposition of waves, with a plethora of applications ranging from the study of atomic and molecular electronic structures [2] to the search for biosignature spectral lines in extrasolar planets [3]. In an FT spectrometer, the intensity variation of the output beam is measured while scanning an interferometer delay line whose longest optical path determines the spectral resolution of the device, with the latest developments [4] reaching a resolution limit of $0.0005 \mathrm{~cm}^{-1}$ as a result of an optical delay of $11.7 \mathrm{~m}$. Resolutions up to $100 \mathrm{kHz}$ have also been demonstrated with frequency comb FT spectrometers [5]. FT spectrometers benefit from a large radiant throughput (étendue), which is an intrinsic property of a Michelson interferometer [6,7] and can be readily combined with extreme laser sources of petawatt-power ultrashort pulses [8], allowing a precise characterization of chemical compounds that could shed light on some current astrophysical problems [9,10]. Further increasing the optical path and henceforth the resolution is intriguing, as it would allow analysis of spectral lines that could not previously be resolved. However, these prospects are challenged by major engineering difficulties and requirements of interferometric stability that must be maintained in a large-scale bulk optical instrument during a prolonged spectrum acquisition time. To overcome these hurdles, here we propose a static spatial heterodyne FT (SHFT) spectrometer with optical fibers as delay elements. As a proof of concept, our device incorporates an array of Mach-Zehnder fiber interferometers with linearly increasing optical path difference, generating a wavelength-dependent stationary interferogram in the spatial domain that can be read in a single shot without scanning elements. The input spectrum is retrieved from the interferogram using spectral inversion algorithms, further allowing us to compensate interferometric deviations from ideal behavior. The extremely low propagation losses of the optical fibers [11] of
$2 \times 10^{-4} \mathrm{~dB} / \mathrm{m}$ and their ability to coil long delay lines in a compact volume, combined with the device advantage of an instantaneous readout, result in an instrument with excellent prospects of being scalable beyond current spectroscopy resolution limits.

The basic concept behind sSHFT spectroscopy is to perform simultaneous interferometric measurements of a single source, each measurement spatially sampling a linearly increasing optical path $[12,13]$. This configuration does not require any moving element, unlike a conventional FT spectrometer, and its resolution is limited by the maximum measured optical path delay, without requiring an interaction between the measured signal and a local oscillator as in coherent spectroscopy [14]. SHFT spectrometers can be implemented as an interferometer array integrated on a photonic chip [15-18], allowing multiple input apertures for an increased radiant throughput compared to planar waveguide devices with a single input waveguide, such as arrayed waveguide gratings [19]. Furthermore, this allows us to individually characterize the transfer function of each interferometer element of the array, and in principle it enables the computational compensation of deviations from the ideal design that may arise from fabrication limitations or imperfections. However, maximum optical path delays in such integrated spectrometer chips are limited to a few centimeters [15-18].

To exploit the advantages of spatial heterodyne spectroscopy while improving its resolution, our FT spectrometer is implemented as an array of $N$ MachZehnder interferometers (MZIs) built with optical fibers, as shown in Fig. 1. The key advantage of the fiber optics configuration is that the extremely low propagation and bending losses of the optical fibers allow substantially longer and more compact optical delays than current free-space bulk optics instruments, therefore allowing us to fold delay lines in a small volume, where a controlled environment is easier to maintain. The length difference between the two optical fiber arms of each 
MZI increases linearly across the interferometer array, hence generating a wavelength-dependent spatial interferogram at the MZI array output. The relation between the input spectral distribution and the output interferogram $\Upsilon\left(x_{i}\right)$ is determined within the free spectral range (FSR) by a cosine FT [1]]:

$$
\Upsilon\left(x_{i}\right)=\int_{0}^{\mathrm{FSR}} B(\bar{\sigma}) \cos 2 \pi \bar{\sigma} x_{i} \mathrm{~d} \bar{\sigma}
$$

where $B$ is the spectral density of the input signal as a function of wavenumber $\sigma$ and $x_{i}$ is the optical path delay of the $i$-th interferometer in the fiber array. When no phase errors are present, the FSR of the device is determined by the periodic presence of a wavelength, at which a monochromatic input produces a constant spatial power distribution at the outputs of the MZI array, at the Littrow wavelength. Within half the range between two Littrow wavelengths, the output patterns of each MZI define the orthogonal base of an invertible linear transformation, and the input spectrum can be unambiguously calculated from the output interferogram. This results in a FSR of half the beat period of the least unbalanced MZI for aliasing-free measurements. The wavelength resolution $(\delta \lambda)$ of the device is directly enhanced by increasing the optical fiber delays, specifically the maximum path difference $L_{\max }$ of the MZI array: $\delta \lambda=\lambda_{0} / L_{\max } n_{g}$, where $\lambda_{0}$ is the device operational central wavelength and $n_{g}$ is the optical fiber group index. Since the whole interferogram is measured in a single shot, the system allows time-resolved measurements.

To experimentally prove this concept, we built an array of 24 MZIs with linearly increasing optical path differences and a maximum interferometer arm imbalance of $24 \mathrm{~cm}$. The 48 input facets of two sets of singlemode polarization-maintaining (PM) fibers were evenly arranged, fixed, and polished in a square ferule holder of $16 \mathrm{~mm}^{2}$, as shown in the inset of Fig. 1. PM fibers were used to prevent polarization mode dispersion in conventional SMF. The collection area can be increased with additional optical fibers, though this is bound by practical size limitations, and with recently developed pitchreducing optical fiber arrays [20]. The bundle was split

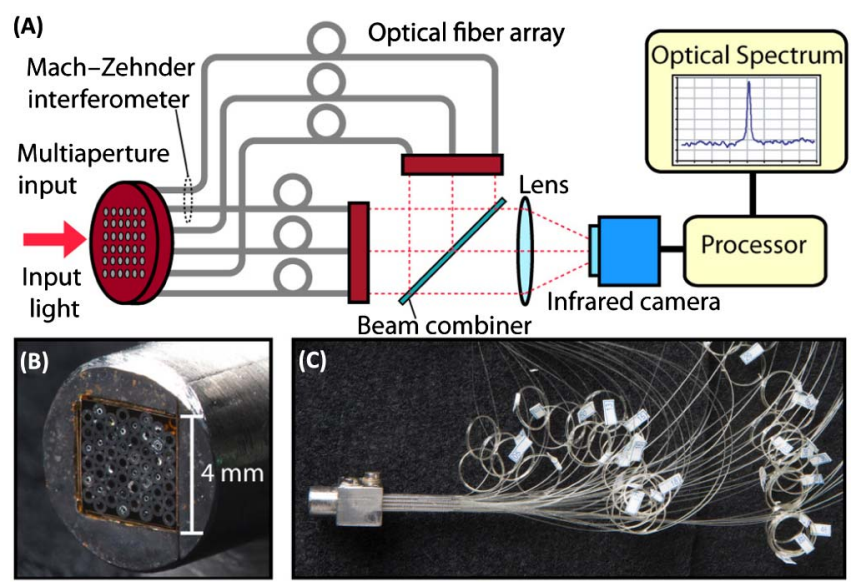

Fig. 1. (A) Schematic of the optical fiber SHFT spectrometer. Input light is collected by a multiaperture input (B) comprising two sets of 24 optical fibers (C). into two sets of 24 fibers configured in pairs, with the outputs of each pair arranged into a rectangular array and combined to form 24 MZIs. The output signals of the array were collected simultaneously in a single shot with a near-IR imaging InGaAs array, and the spectrum was calculated using a retrieval algorithm developed inhouse. Beam combination and coupling to the imaging array could also be implemented with optical fibers to exclude free-space elements.

Each interferometer of the fabricated device was individually characterized in a $100 \mathrm{pm}$ range starting at $1550 \mathrm{~nm}$ with a high-resolution tunable continuous wave laser with a sampling wavelength step of $100 \mathrm{fm}$. The measured calibration map in Fig. 2 shows the experimental transmittance function of each MZI in the wavelength range under analysis. The intrinsically low loss of the optical fibers provided a fringe contrast close to the $100 \%$ theoretical maximum even for the most unbalanced MZIs. The experimental spectral response of the MZIs of the array showed phase and amplitude errors from the ideal sinusoidal MZI spectrum, caused by fabrication deviations and other experimental interference, such as Fabry-Perot cavity effects and ambient fluctuations. These deviations result in a degeneration of the orthogonality of the transformation base and prevent the use of classical FT spectral retrieval algorithms without active compensation of phase errors [21]. Instead, our spectral retrieval technique is based on a system of linear equations defined by the MZI transmittance functions. The spectral response of each interferometer was calibrated within the aliasing-free FSR and sampled at $M$ equidistant wavelengths to define an $N \times M$ transformation matrix $T$ that comprises the information of the spectral dependence of the output interferograms, including any phase and amplitude deviations from an ideal performance. In order to retrieve the spectrum associated with the interferogram of an arbitrary input signal, the interferogram is multiplied by the pseudoinverse of the transformation matrix, computed by single value

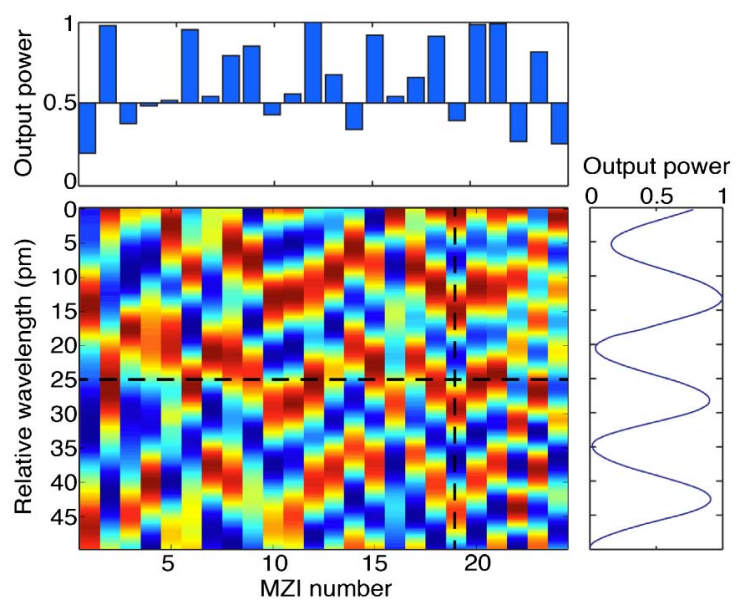

Fig. 2. Calibration map of the fabricated optical fiber SHFT spectrometer in a FSR of $50 \mathrm{pm}$ starting at $1550 \mathrm{~nm}$. The rows of the matrix represent the normalized power of the output interferogram for each wavelength (top), whereas the columns comprise the spectral response of each interferometer (right). 


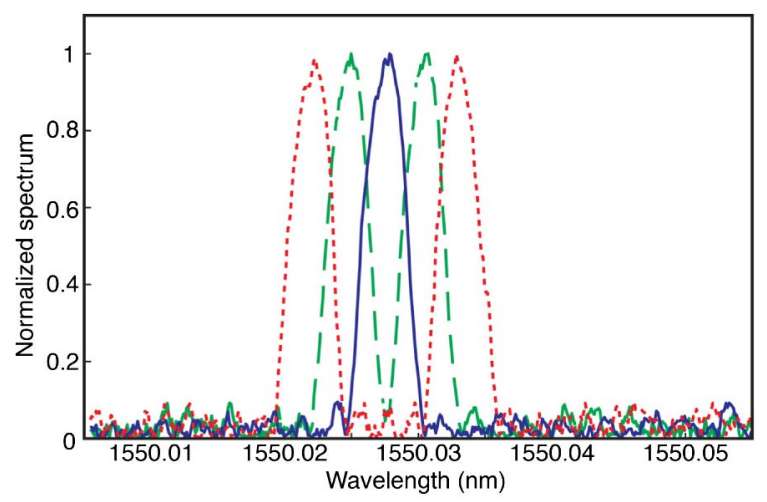

Fig. 3. Spectra of a single narrowband laser source (solid) and two doublets of two monochromatic lines separated $5.5 \mathrm{pm}$ (dashed) and $11 \mathrm{pm}$ (dotted), retrieved from experimental measurements with the optical fiber SHFT spectrometer. A FWHM resolution of $3 \mathrm{pm}$ is demonstrated.

decomposition [22], achieving a high degree of phase and amplitude error compensation.

The spectral retrieval performed with our optical fiber array SHFT spectrometer is demonstrated in Fig. 3, showing the spectral density measurement of a single monochromatic source (solid), and doublets of two monochromatic lines separated by $5.5 \mathrm{pm}$ (dotted) and $11 \mathrm{pm}$ (dashed), respectively. Each spectral line is shown to have a broadening of $3 \mathrm{pm}$, measured at the full width at half-maximum (FWHM). Hence a resolution of $0.0125 \mathrm{~cm}^{-1}$ is demonstrated within an FSR of $50 \mathrm{pm}$.

This first demonstration of an optical fiber SHFT spectrometer represents a significant milestone toward pushing current resolution boundaries of passive FT spectrometers. Low-loss optical fibers readily allow implementing of MZIs with physical length differences of the order of kilometers while preserving good SNR and compact instrument size. Since the resolution of the device is determined by the optical path difference of the most unbalanced MZI, this would result in an extreme resolving power. As an example, an MZI array with a maximum path length difference of $1 \mathrm{~km}$, working with the demonstrated spectral retrieval technique, would enable measuring of spectral lines with a $0.7 \mathrm{fm}$ FWHM broadening, that is, an unprecedented spectroscopic resolution of $3 \times 10^{-6} \mathrm{~cm}^{-1}$. Nevertheless, to obtain such an astonishing spectral resolution based on optical fiber MZI arrays, while still minimizing phase fluctuations, significant stability and calibration requirements need to be overcome. A possible solution may include the continuous active monitoring of each interferometer with stabilized single-frequency lasers. The proposed SHFT spectrometer can be advantageously used in combination with extreme light sources of radiation [8], which provide ultrashort pulses (10-15 fs) with a power up to $10 \mathrm{PW}$. This combination could allow us to identify and measure the spectral characteristics of highly excited products of chemical or physical processes in a high-energy laser spark. The instantaneous readout of the MZI array interferometric pattern also enables time-resolved measurements of these processes.

This work demonstrates a SHFT spectrometer based on optical fiber delays. The demonstrated SHFT spectrometer concept makes it possible to scale up the optical delays as required to reach unprecedented resolutions and to potentially resolve spectral lines that have not been previously observed.

Financial support from the National Research Council of Canada and Spanish Ministry of Economy (TEC200804105 and TEC2011-23629) is acknowledged.

\section{References}

1. P. R. Griffiths and J. A. de Haseth, Fourier Transform Infrared Spectrometry (Wiley, 2007).

2. N. De Oliveira, M. Roudjane, D. Joyeux, D. Phalippou, J. C. Rodier, and L. Nahon, Nat. Photonics 5, 149 (2011).

3. E. Schwartz, S. G. Lipson, and E. N. Ribak, Astron. J. 144, 71 (2012).

4. S. Albert, K. K. Albert, and M. Quack, Handbook of High-Resolution Spectroscopy (Wiley, 2011).

5. J. Mandon, G. Guelachvili, and N. Picqué, Nat. Photonics 3, 99 (2009).

6. A. A. Michelson and E. Morley, Am. J. Sci. 34, 333 (1887).

7. P. Jacquinot, J. Opt. Soc. Am. 44, 761 (1954).

8. G. A. Mourou, G. Korn, W. Sandner, and J. L. Collier, Extreme Light Infrastructure Whitebook (THOSS Media $\mathrm{GmbH}, 2011)$.

9. S. Albert, K. K. Albert, P. Lerch, and M. Quack, Faraday Discuss. 150, 71 (2011).

10. T. Wilken, G. Lo Curto, R. A. Probst, T. Steinmetz, A. Manescau, L. Pasquini, J. I. G. Hernández, R. Rebolo, T. W. Hänsch, T. Udem, and R. Holzwarth, Nature 485, 611 (2012).

11. Corning SMF-28e+ LL Optical Fiber, 2011, http://www . corning.com/WorkArea/showcontent. . sppx?id=49145.

12. P. Cheben, I. Powell, S. Janz, and D.-X. Xu, Opt. Lett. 30, 1824 (2005).

13. J. M. Harlander, F. L. Roesler, J. G. Cardon, C. R. Englert, and R. R. Conway, Appl. Opt. 41, 1343 (2002).

14. J. Pelayo, F. Villuendas, C. D. Heras, and E. Pellejer, IEEE Photon. Technol. Lett. 17, 855 (2005).

15. M. Florjańczyk Miroslaw, P. Cheben, S. Janz, A. Scott, B. Solheim, and D.-X. Xu, Opt. Express 15, 18176 (2007).

16. K. Okamoto, H. Aoyagi, and K. Takada, Opt. Lett. 35, 2103 (2010).

17. M. Florjańczyk, C. Alonso-Ramos, P. Bock, A. Bogdanov, P. Cheben, Í. Molina-Fernández, S. Janz, B. Lamontagne, A. Ortega-Moñux, A. Scott, K. Sinclair, B. Solheim, and D.-X. Xu, Opt. Quantum Electron. 44, 549 (2012).

18. A. V. Velasco, P. Cheben, P. J. Bock, A. Delâge, J. H. Schmid, J. Lapointe, S. Janz, M. L. Calvo, D.-X. Xu, M. Florjańczyk, and M. Vachon, Opt. Lett. 38, 706 (2013).

19. P. Cheben, J. H. Schmid, A. Delâge, A. Densmore, S. Janz, B. Lamontagne, J. Lapointe, E. Post, P. Waldron, and D.-X. Xu, Opt. Express 15, 2299 (2007).

20. V. I. Kopp and A. Z. Genack, Nat. Photonics 5, 470 (2011).

21. K. Takada, H. Aoyagi, and K. Okamoto, Opt. Lett. 36, 1044 (2011).

22. G. H. Golub and C. Reinsch, Numer. Math. 14, 403 (1970). 